\title{
DETERMINATION OF CONDUCTIVITY IN A HEAT EQUATION
}

\author{
PING WANG and KEWANG ZHENG
}

(Received 1 February 2000)

\begin{abstract}
We consider the problem of determining the conductivity in a heat equation from overspecified non-smooth data. It is an ill-posed inverse problem. We apply a regularization approach to define and construct a stable approximate solution. We also conduct numerical simulation to demonstrate the accuracy of our approximation.
\end{abstract}

Keywords and phrases. Heat equation, inverse problem, regularization.

2000 Mathematics Subject Classification. Primary 35R25, 35R30; Secondary 35K05.

1. Introduction. The problem of determining the conductivity $a(t)$ in

$$
u_{t}(x, t)=a(t) u_{x x}(x, t), \quad 0<x<1,0<t<T
$$

from overspecified smooth data has been studied by many people. For example, Jones in [4] proved existence and uniqueness of the solution of the inverse problem. Douglas and Jones provided in [3] numerical approach for determining the unknown coefficient. Around the same time, Cannon in [1] gave a different approach to the same problem. Later, in [2], Cannon considered (1.1) in which the conductivity was assumed to be an unknown constant and the heat flow rate was measured only for a single time.

For practical reasons, it is more interesting to consider the problem of determining the unknown conductivity coefficient from non-smooth data. In such case, the problem is ill posed, as we will demonstrate later. New way of determining the unknown coefficient is needed.

In this paper, we consider the problem of determining $a(t)$ in the following parabolic problem:

$$
\begin{aligned}
u_{t}(x, t) & =a(t) u_{x x}(x, t), \quad 0<x, 0<t<T ; \\
u(x, 0) & =0, \quad 0<x ; \\
u(0, t) & =f(t), \quad 0<t<T, f(0)=0 ; \\
-u_{x}(0, t) & =g_{0}, \quad 0<t<T,
\end{aligned}
$$

where $u(x, t)$ and $a(t)$ are unknown and to be determined from known non-smooth data $f(t), g_{0}$ ( $g_{0}$ is a positive constant).

Let us first exam the ill-posedness of the problem.

For smooth function $f(t)$, if $u(x, t)$ is a classical solution of (1.2), then it can be shown that

$$
u(x, t)=\frac{g_{0}}{\sqrt{\pi}} \int_{0}^{t} \frac{a(\tau)}{\sqrt{\theta(t)-\theta(\tau)}} e^{-x^{2} /(4(\theta(t)-\theta(\tau)))} d \tau,
$$




$$
\theta(t)=\int_{0}^{t} a(\tau) d \tau .
$$

Letting $x$ approach zero, we obtain from (1.3) that

$$
f(t)=\frac{g_{0}}{\sqrt{\pi}} \int_{0}^{t} \frac{a(\tau)}{\sqrt{\theta(t)-\theta(\tau)}} d \tau,
$$

which gives immediately

$$
\begin{aligned}
& \theta(t)=\frac{\pi}{4 g_{0}^{2}} f^{2}(t), \\
& a(t)=\frac{\pi}{2 g_{0}^{2}} f(t) f^{\prime}(t) .
\end{aligned}
$$

The above computations are possible only under the assumption that the boundary term $f(t)$ is smooth.

In practice, unfortunately, the known data $f(t)$ (like boundary temperature) is obtained experimentally. It is generally not a smooth function in time. Then it is not possible to solve $a$ in (1.4) in the classical sense.

Even if the data is obtained in such a way that the classical solution of (1.4) exists, this solution may not depend on the data continuously. To see this, we consider the following example.

EXAMPLE 1.1. Let $g_{0}=\sqrt{\pi / 2}$ and

$$
f_{T}(t)= \begin{cases}t & 0 \leq t \leq \frac{1}{2}, \\ \left(2 t-t^{2}-\frac{1}{2}\right)^{1 / 2} & \frac{1}{2}<t \leq 1 .\end{cases}
$$

It is easy then to obtain the exact solution of (1.4) as

$$
a_{T}(t)= \begin{cases}t & 0 \leq t \leq \frac{1}{2} \\ 1-t & \frac{1}{2}<t \leq 1\end{cases}
$$

Now take an approximate data function $f_{\delta}=f_{T}+(\pi / 4)^{1 / 4} \delta \sin \left(t / \delta^{3}\right)$. While one has

$$
\left\|f_{\delta}-f_{T}\right\|_{L^{4}} \leq \delta^{4}
$$

the difference between the solutions

$$
\left\|a_{\delta}(t)-a_{T}(t)\right\|_{C} \geq \frac{\pi}{4 \delta},
$$

which shows that the problem of determining $a(t)$ in $C$ from boundary data in $L^{4}$ is ill posed.

In this paper, we will apply a regularization method (cf. $[5,6,7])$ to define and construct a stable solution of (1.4), which is sometimes referred as a mapping:

$$
A[a]=\int_{0}^{t} a(\tau) d \tau=\theta(t),
$$


where $\theta$ is given in (1.6). We will also conduct numerical computations to verify the accuracy of our approximate approach.

2. Regularizing operator. Define the following functional:

$$
M^{\alpha}[a, \theta]=\int_{0}^{T}\left[\int_{0}^{t} a(\tau) d \tau-\theta(t)\right]^{2} d t+\alpha \int_{0}^{T}\left[a^{2}(\tau)+a^{\prime}(\tau)^{2}\right] d \tau .
$$

THEOREM 2.1. For every $\theta(t)$ in $L^{2}[0, T]$ and every positive number $\alpha$, there exists a unique function $a_{\alpha}(t) \in C[0, T]$ that minimizes the functional (2.1).

Proof. Considering the first variation of the functional (2.1), we can see the minimizer of the functional is the solution of the following Euler integrodifferential equation:

$$
\alpha\left(a^{\prime \prime}-a\right)=\int_{\tau}^{T} \int_{0}^{t} a(\xi) d \xi d t-\int_{T}^{T} \theta(t) d t
$$

subject to the boundary conditions $a(0)=0, a(T)=0$. It is trivial to show that there exists a unique solution of (2.2). We omit the details.

Based on Theorem 2.1, we now define an operator $R(\theta, \alpha)$ from the set of pairs: $(\theta, \alpha)$, where $\theta \in L^{2}, \alpha>0$, to the space $C[0, T]$ so that the element $a_{\alpha}=R(\theta, \alpha)$ minimizes the functional $M^{\alpha}$. In what follows, we need to show that, for an appropriate $\alpha$, $a_{\alpha}$ is a stable approximate solution of (1.4), namely, $R(\theta, \alpha)$ is a regularizing operator.

THEOREM 2.2. Let $a_{T}$ denote a solution of (1.4) with right-hand member $\theta_{T}$ and $a_{\alpha}=R\left(\theta_{\delta}, \alpha\right)$, where $\delta$ measures the error between $\theta_{T}$ and $\theta_{\delta}$. For any positive number $\varepsilon$, there exists a number $\delta(\varepsilon)>0$, such that the inequality

$$
\left\|\theta_{\delta}-\theta_{T}\right\|_{L^{2}} \leq \delta \leq \delta(\varepsilon)
$$

implies the inequality

$$
\left\|a_{\alpha}-a_{T}\right\|_{C} \leq \varepsilon
$$

where $\alpha=\alpha(\delta)=\delta^{\lambda}, 0<\lambda \leq 2$.

Proof. Since $a=a_{\alpha}$ is the minimizer of functional $M^{\alpha}\left[a, \theta_{\delta}\right]$, we have

$$
M^{\alpha}\left[a_{\alpha}, \theta_{\delta}\right] \leq M^{\alpha}\left[a_{T}, \theta_{\delta}\right]
$$

Therefore,

$$
\begin{aligned}
\alpha \int_{0}^{T}\left(a_{\alpha}^{2}(\tau)+\left(a_{\alpha}^{\prime}(\tau)\right)^{2}\right) d \tau \leq & \int_{0}^{T}\left(\int_{0}^{t} a_{T}(\tau) d \tau-\theta_{\delta}(t)\right)^{2} d t \\
& +\alpha \int_{0}^{T}\left(a_{T}^{2}(\tau)+\left(a_{T}^{\prime}(\tau)\right)^{2}\right) d \tau \\
\leq & \delta^{2}+\alpha \int_{0}^{T}\left(a_{T}^{2}(\tau)+\left(a_{T}^{\prime}(\tau)\right)^{2}\right) d \tau \leq \delta^{2} d,
\end{aligned}
$$


where $d=1+\int_{0}^{T}\left(a_{T}^{2}(\tau)+\left(a_{T}^{\prime}(\tau)\right)^{2}\right) d \tau$. Thus,

$$
\int_{0}^{T}\left(a_{\alpha}^{2}(\tau)+\left(a_{\alpha}^{\prime}(\tau)\right)^{2}\right) d \tau \leq d, \quad \int_{0}^{T}\left(a_{T}^{2}(\tau)+\left(a_{T}^{\prime}(\tau)\right)^{2}\right) d \tau \leq d .
$$

Consequently, the elements $a_{\alpha}, a_{T}$ belong to the following compact subset of space $C[0, T]$ :

$$
F=\left\{a(\tau): \int_{0}^{T}\left(a^{2}(\tau)+\left(a^{\prime}(\tau)\right)^{2}\right) d \tau \leq d\right\} .
$$

Since the mapping $A: F \rightarrow A F$ ( $A$ is defined in (1.12)) is continuous and one-to-one, the inverse mapping $A^{-1}: A F \rightarrow F$ is also continuous. It means that, for arbitrary $\varepsilon>0$, there exists a number $\gamma(\varepsilon)>0$ such that the inequality

$$
\left\|\theta_{\alpha}-\theta_{T}\right\|_{L^{2}} \leq \gamma(\varepsilon), \quad \theta_{\alpha}=A\left(a_{\alpha}\right), \theta_{T}=A\left(a_{T}\right) \in A F
$$

implies the inequality

$$
\left\|a_{\alpha}-a_{T}\right\|_{C[0, T]} \leq \varepsilon .
$$

On the other hand, for $\theta_{\delta}, \theta_{\alpha}$, we have

$$
\begin{aligned}
\left\|\theta_{\alpha}-\theta_{\delta}\right\|_{L^{2}}^{2} & =\int_{0}^{T}\left(\int_{0}^{t} a_{\alpha}(\tau) d \tau-\theta_{\delta}(t)\right)^{2} d t \\
& \leq M^{\alpha}\left[a_{\alpha}, \theta_{\delta}\right] \leq M^{\alpha}\left[a_{T}, \theta_{\delta}\right] \leq \delta^{\lambda} d .
\end{aligned}
$$

Obviously,

$$
\left\|\theta_{\alpha}-\theta_{T}\right\|_{L^{2}} \leq\left\|\theta_{\alpha}-\theta_{\delta}\right\|_{L^{2}}+\left\|\theta_{\delta}-\theta_{T}\right\|_{L^{2}},
$$

which implies that

$$
\left\|\theta_{\alpha}-\theta_{T}\right\|_{L^{2}} \leq \delta^{\lambda / 2} \sqrt{d}+\delta \leq \delta^{\lambda / 2}(1+\sqrt{d}) .
$$

To end the proof of the theorem, let

$$
\delta(\varepsilon)=\left(\frac{\gamma(\varepsilon)}{1+\sqrt{d}}\right)^{2 / \lambda} .
$$

Theorem 2.2 shows that $a_{\alpha}$ can be taken as an approximate solution of (1.4) with approximate right-hand member $\theta=\theta_{\delta}$.

Next, we need to show that $\theta$ depends on $f, g_{0}$ continuously.

THEOREM 2.3. Suppose that $\left\|f_{\delta}-f_{T}\right\|_{L^{4}[0, T]} \leq \delta,\left|g_{\delta}-g_{0}\right| \leq \delta$, then

$$
\left\|\theta_{\delta}-\theta_{T}\right\|_{L^{2}} \leq D \delta
$$

where

$$
D=\frac{4 \pi}{g_{0}^{3}}\left\|f_{T}\right\|_{L^{4}}\left(8\left(g_{0}^{4}+\left\|f_{T}\right\|_{L^{4}}^{4}\right)\right)^{1 / 4}
$$


Proof. From (1.6) and Cauchy inequality,

$$
\begin{aligned}
\left\|\theta_{\delta}-\theta_{T}\right\|_{L^{2}}^{2} & =\left(\frac{\pi}{4 g_{0}^{2} g_{\delta}^{2}}\right)^{2} \int_{0}^{T}\left(g_{0}^{2} f_{\delta}^{2}(t)-g_{d} t^{2} f_{T}^{2}(t)\right)^{2} d t \\
& =\left(\frac{\pi}{4 g_{0}^{2} g_{\delta}^{2}}\right)^{2} \int_{0}^{T}\left(g_{0} f_{\delta}(t)-g_{\delta} f_{T}(t)\right)^{2}\left(g_{0} f_{\delta}(t)+g_{\delta} f_{T}(t)\right)^{2} d t \\
& \leq\left(\frac{\pi}{g_{0}^{4}}\right)^{2}\left(\int_{0}^{T}\left(g_{0} f_{\delta}(t)-g_{\delta} f_{T}(t)\right)^{4} d t\right)^{1 / 2} \cdot\left(\int_{0}^{T}\left(g_{0} f_{\delta}(t)+g_{\delta} f_{T}(t)\right)^{4} d t\right)^{1 / 2},
\end{aligned}
$$

where $g_{0} \leq 2 g_{\delta}$. The result in this theorem follows from the following estimates:

$$
\begin{aligned}
\int_{0}^{T}\left(g_{0} f_{\delta}(t)+g_{\delta} f_{T}^{(t)}\right)^{4} d t & \leq 8\left(g_{0}^{4}\left\|f_{\delta}\right\|_{L^{4}}^{4}+g_{\delta}^{4}\left\|f_{T}\right\|_{L^{4}}^{4}\right) \\
& \leq 8^{2} g_{0}^{4}\left(\left\|f_{\delta}-f_{T}\right\|_{L^{4}}^{4}+3\left\|f_{T}\right\|_{L^{4}}^{4}\right) \quad\left(g_{\delta}<2 g_{0}\right) \\
& \leq 8^{2} g_{0}^{4}\left(\delta^{4}+3\left\|f_{T}\right\|_{L^{4}}^{4}\right) \\
& \leq 8^{2} 4 g_{0}^{4}\left\|f_{T}\right\|_{L^{4}}^{4} \quad\left(\delta<\left\|f_{T}\right\|_{L^{4}}^{4}\right), \\
\int_{0}^{T}\left(g_{0} f_{\delta}(t)-g_{\delta} f_{T}^{(t)}\right)^{4} d t & =\int_{0}^{T}\left(g_{0}\left(f_{\delta}(t)-f_{T}(t)\right)+\left(g_{0}-g_{d} t\right) f_{T}^{(t)}\right)^{4} d t \\
& \leq 8\left(g_{0}^{4}\left\|f_{\delta}-f_{T}\right\|_{L^{4}}^{4}+\left|g_{0}-g_{\delta}\right|^{4}\left\|f_{T}\right\|_{L^{4}}^{4}\right) \\
& \leq 8\left(g_{0}^{4}+\left\|f_{T}\right\|_{L^{4}}^{4}\right) \delta^{4} .
\end{aligned}
$$

Combing Theorems 2.2 and 2.3, we have the following stability result.

THEOREM 2.4. Given $\varepsilon>0$, there exists $\delta>0, \alpha=\alpha(\delta)$, such that, for the approximate solution $a_{\alpha}$ corresponding to $f_{\delta}, g_{\delta}$ and the exact solution $a_{T}$ corresponding to $f_{T}, g_{0}$, inequalities

$$
\left\|f_{\delta}-f_{T}\right\|_{L^{4}[0, T]} \leq \delta, \quad\left|g_{\delta}-g_{0}\right| \leq \delta
$$

imply

$$
\left\|a_{\alpha}-a_{T}\right\|_{C} \leq \varepsilon
$$

Therefore, $a_{\alpha}$ can be taken as a stable approximate solution of (1.2).

3. A numerical example. To demonstrate the applicability of our approximation approach, we consider the example in Section 2. Replacing the Euler integral equation (2.2) by its finite difference approximation on a uniform grid with step $h=$ $T /(n+1), T=1$, we obtain the following system of linear equations:

$$
\alpha\left[\frac{a_{j+1}-2 a_{j}+a_{j-1}}{h^{2}}-a_{j}\right]=h^{2} \sum_{i=j}^{n} \sum_{k=1}^{i} a_{k}-h \sum i=j^{n} \theta_{i}, \quad j=1, \ldots, n,
$$

with $a_{0}=a_{n+1}=0, a_{j}=a\left(\tau_{j}\right), \theta_{i}=\theta\left(t_{i}\right)$. 
Take the regularization parameter $\alpha$ in the form of

$$
\alpha=\alpha(\delta)=(D \delta)^{\lambda},
$$

where $0<\lambda \leq 2, D$ is given by (2.16). With $\left(\theta_{\delta}\right)_{i}=\theta_{\delta}\left(t_{i}\right)=\left(\pi / g_{\delta}^{2}\right) f_{\delta}\left(t_{i}\right)^{2}, g_{\delta}=g_{0}+$ $\delta$, we want to recover $a_{T}$ from (3.1). The numerical comparison between the exact solution $a_{T}$ and the approximate solution are given in the following table $(n+79$, $\left.\delta=10^{-8}, \lambda=1.84\right)$ :

\begin{tabular}{c|lllllll}
\hline$t$ & 0.025 & 0.05 & 0.075 & 0.1 & 0.125 & 0.15 & 0.175 \\
\hline$a_{T}(t)$ & 0.025 & 0.05 & 0.075 & 0.1 & 0.125 & 0.15 & 0.175 \\
\hline$a_{\alpha}(t)$ & 0.025005 & 0.050030 & 0.074908 & 0.0100105 & 0.125084 & 0.149973 & 0.175053 \\
\hline \hline$t$ & 0.2 & 0.225 & 0.25 & 0.275 & 0.3 & 0.325 & 0.35 \\
\hline$a_{T}(t)$ & 0.2 & 0.225 & 0.25 & 0.2750 & 0.3 & 0.325 & 0.35 \\
\hline$a_{\alpha}(t)$ & 0.199973 & 0.225043 & 0.250111 & 0.275142 & 0.300033 & 0.325053 & 0.350123 \\
\hline \hline$t$ & 0.375 & 0.4 & 0.425 & 0.45 & 0.475 & 0.5 & \\
\hline$a_{T}(t)$ & 0.375 & 0.4 & 0.425 & 0.45 & 0.475 & 0.5 & \\
\hline$a_{\alpha}(t)$ & 0.374837 & 0.400034 & 0.425039 & 0.449937 & 0.45057 & 0.493663 & \\
\hline
\end{tabular}

One can see that, unlike the discussion in Section 2, our approximate solution $a_{\alpha}$ and the exact solution $a_{T}$ match very well. This shows that the regularization approach discussed in this work is an effective way of determining the unknown conduction coefficient $a(t)$ in the heat equation (1.2).

\section{REFERENCES}

[1] J. R. Cannon, Determination of an unknown coefficient in a parabolic differential equation, Duke Math. J. 30 (1963), 313-323. MR 28\#358. Zbl 117.06901.

[2] - Determination of certain parameters in heat conduction problems, J. Math. Anal. Appl. 8 (1964), 188-201. MR 28\#3261. Zbl 131.32104.

[3] J. Douglas, Jr. and B. F. Jones, Jr., The determination of a coefficient in a parabolic differential equation. II. Numerical approximation, J. Math. Mech. 11 (1962), 919-926. MR 27\#3949. Zbl 112.32603.

[4] B. F. Jones, Jr., The determination of a coefficient in a parabolic differential equation. I. Existence and uniqueness, J. Math. Mech. 11 (1962), 907-918. MR 27\#3948. Zbl 112.32602.

[5] Z. Kewang and P. Wang, Numerical approximation of an unknown boundary term in a heat equation, Neural Parallel Sci. Comput. 2 (1994), no. 4, 451-457. CMP 1303299. Zbl 815.65130.

[6] P. Wang and K. Zheng, Determination of unknown term in a heat equation, Arab. J. Sci. Eng. Sect. C Theme Issues 22 (1997), no. 2, 149-157. CMP 1617 038. Zbl 908.65122.

[7] __ Regularization of an Abel equation, Integral Equations Operator Theory 29 (1997), no. 2, 243-249. MR 98i:45004. Zbl 891.65137.

Ping Wang: Department of Mathematics, Pennsylvania State University, Schuylkill HAVEN, PA 17972, USA

Kewang Zheng: Department of Mathematics, Hebei University of SCience and TechNOLOGY, CHINA 


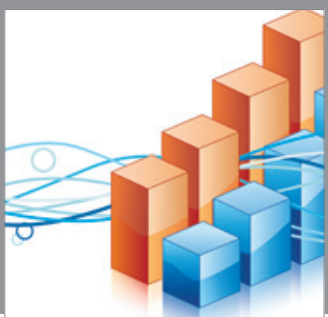

Advances in

Operations Research

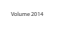

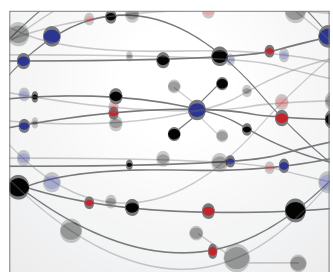

\section{The Scientific} World Journal
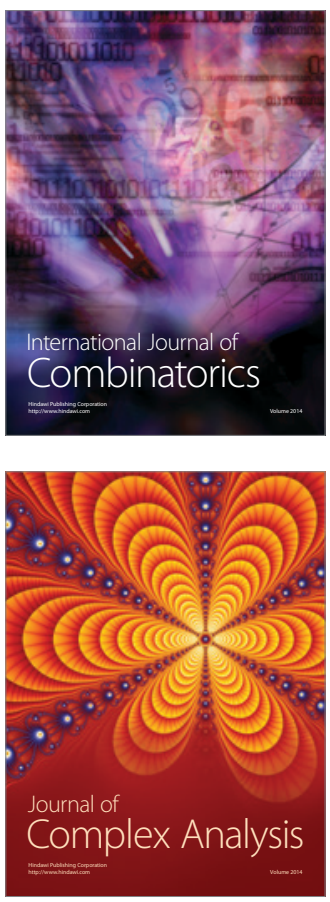

International Journal of

Mathematics and

Mathematical

Sciences
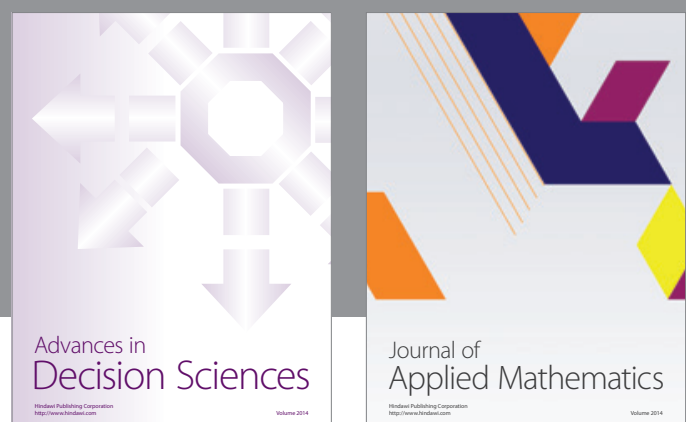

Journal of

Applied Mathematics
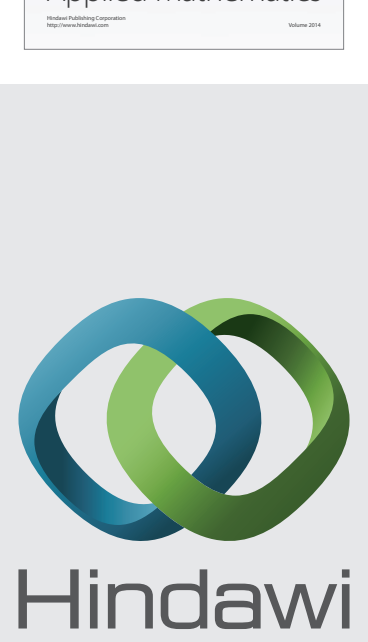

Submit your manuscripts at http://www.hindawi.com
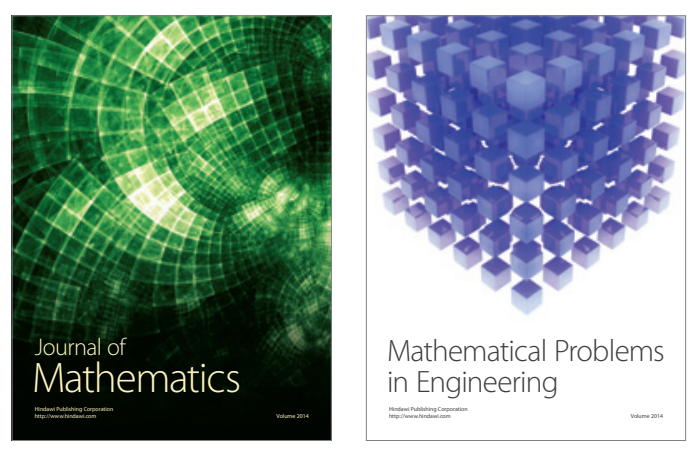

Mathematical Problems in Engineering
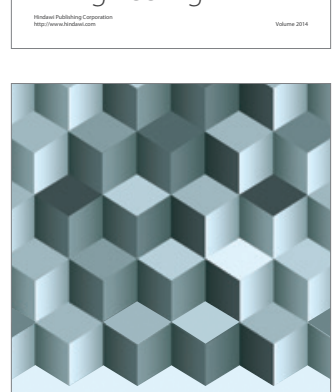

Journal of

Function Spaces
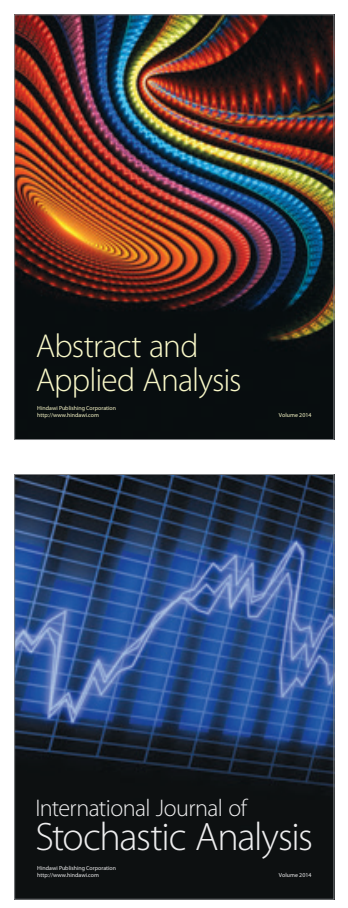

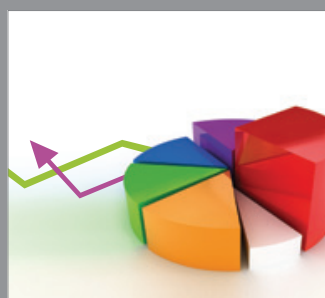

ournal of

Probability and Statistics

Promensencen
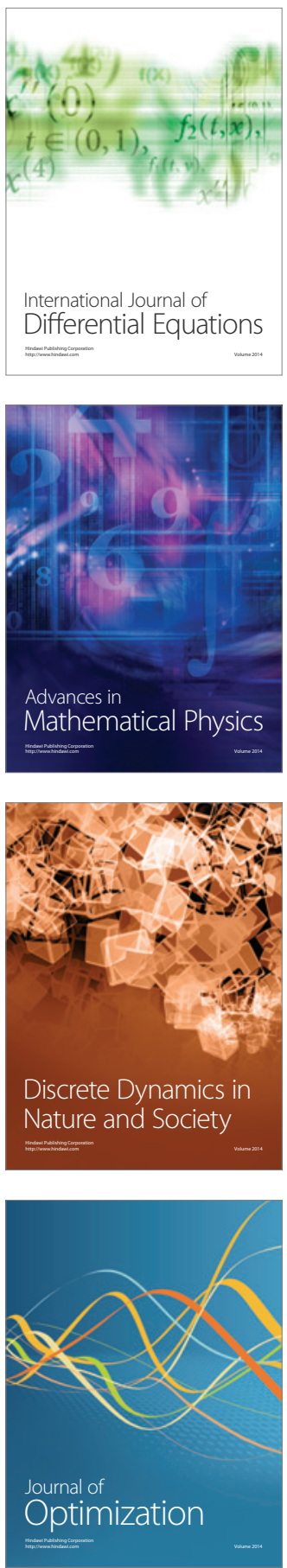